\title{
A GIS framework for the assessment of tick impact on human health in a changing climate
}

\author{
Agustin Estrada-Peña, José M. Venzal \\ Department of Parasitology, Faculty of Veterinary Medicine, University of Zaragoza, Zaragoza, Spain
}

\begin{abstract}
A framework to evaluate the impact of ticks on human health under various scenarios of climate change is proposed. The purpose is not to provide a comprehensive plan (e.g. the economic impact of ticks on human society is not included), instead we wish to describe a series of indices that would be helpful by obtaining homogeneous comparisons of impact and vulnerability exerted by ticks in different regions, countries or continents, using normalized sets of population, vegetation, climate and physical attributes of the territory. Three tick species, i.e. Dermacentor marginatus, Rhipicephalus turanicus and Hyalomma marginatum, have been traced over the territory of Spain to further explain the computation of these indices. The discussion is based on tick habitat suitability, used as a measure of the abiotic (climate) fitness of the habitat for the species in question, and the sensitivity of each tick species to the rate of change of habitat suitability with respect to climate change. The impact is the rate of change in habitat suitability weighted with a fuzzy logic function evaluating the total number of people in an area, percent of rural population and accessibility of the geographical divisions (expressed as hexagons with a $10 \mathrm{~km}$ radius) used in the study. The different climate scenarios evaluated in relation to ticks show that the north-western part of Spain would suffer the greatest impact in case the mean temperature would increase, while the Mediterranean region would suffer the highest impact if temperatures decreased. Vulnerability, based on the sanitary structure of the territory and on the impact on human activities due to the change in tick distribution and abundance, is proposed as a measure of adaptation of society to these climate scenarios. The cost is evaluated as a function of land use and tick habitat suitability in a buffer zone surrounding each geographic division. All indices proposed have been obtained by search of common and/or publicly available data sets.
\end{abstract}

Keywords: ticks, tick-borne disease, tick impact, society, vulnerability, human health, climate change.

\section{Introduction}

The perceived knowledge today is that advances in technology minimize the negative effects of individual infectious or parasitic diseases and will thus provide much of the short-term protection needed. However, zoonoses present a continuing challenge to human well-being and are particularly associated

Corresponding author:

Agustin Estrada-Peña

Department of Parasitology

Faculty of Veterinary Medicine

University of Zaragoza

Miguel Servet 177, 50013 Zaragoza, Spain

Tel. +34 9767615 558; Fax +34 976761612

E-mail: aestrada@unizar.es with human lifestyles (work and leisure) which bring people into close contact with animals (Polley, 2005). Since humans with increasing frequency invade the various ecological niches of animals, the need to better understand the ecology of parasites of free-ranging wildlife has never been greater. The emergence or resurgence (possibly due to increased reporting) of arthropod-transmitted diseases, has contributed to an increased awareness of the role played by vectors with respect to the transmission of human diseases. As a consequence, arthropods such as mosquitoes and ticks, are increasingly attracting the attention of scientists involved in issues related to human health. While mosquito-transmitted diseases, for example malaria, are important and have received much attention lately, we need to also 
know about the epidemiology of the many serious tick-borne diseases threatening us (Rogers and Randolph, 2000).

Spatial epidemiology consist of the description and analysis of the geographic variation in diseases with respect to demographic, environmental, behavioural, socioeconomic, and infectious risk factors. Recent advances in data availability and analytic methods have created new opportunities for investigators to improve our knowledge of the new challenges to human health (Hay et al., 2000). There is currently much interest in conducting spatial analyses of the pressure exerted by arthropods versus human health and how transmission of arthropod-borne diseases develop under different climate conditions. These analyses have long been recognized in the literature as playing a specific and important role in the description and analysis of patterns of disease as they can highlight sources of heterogeneity or suggest important clues to health determinants.

In the case of ticks, geographic mapping of human diseases may be used to identify possible clusters or areas where ticks are particularly prevalent to define and monitor epidemics, to provide baseline data on tick prevalence, and to show changes in tick abundance patterns over time. To mitigate the risks with regard to tick-borne diseases we must first understand the factors driving the patterns of human-tick contact, then collect variables allowing the generation of models involving large areas such as whole countries. These models should ideally be as general as possible and efforts should be made to incorporate all factors needed to understand the basics of the ecology of the parasites and the rules governing their contact with humans. At the same time they must be highly fine-tuned, built from solid foundations, and firmly based on the ecology of the parasites in question.

What is needed now is a further analysis of a general framework for the computation of the impact on, and the vulnerability of, human populations in relation to ambient tick prevalence under different climate scenarios. We are still far from producing a full-fledged model for the tick-borne diseases, but a first step in that direction would be to move towards a holistic approach to risk assessment of ticks under global climate change. To achieve this we need to combine the various approaches developed by the different communities into a comprehensive risk assessment framework. While some existing references are exercises in definition of terms (i.e. Sutherst, 2004), this study intends to use geographical information systems (GIS) as a procedural skeleton since it can be applied to entire countries (even continents) using the same methodology and the same variables. To that end, a format which would be valid independently of the socioeconomic, behavioural and demographic factors at the site of application is proposed. The advantage of such a framework is that it would be applicable in regions with different ecologies and thus capable to provide homogeneous and comparable estimates of impact and vulnerability.

Framework to compute the impact by ticks on humans under changing climate conditions

\section{Computation of habitat suitability for ticks}

Habitat suitability (HS) is a measure of the contribution of the abiotic component for the survival and permanent colonization of a tick population with regard to its target species. This calculation ignores the biotic component (hosts, competition with other tick species, etc.) that may have an influence on the tick life cycle and persistence at a given site. Furthermore, HS is independent of exposure. There is a misleading practice of considering risks in the absence of a societal response which can result in an exaggerated picture of the perceived risks. Methods to describe HS are based on the environmental niche according to Hutchinson, i.e. an n-dimensional hyper-volume describing the climate conditions with which the range of tick distribution is associated, a concept discussed in detail with respect to ticks in a recent publication (Estrada-Peña et al., 2006). Ecological requirements for a species 
can be conceptualized via the idea of an ecological niche or the set of conditions under which a species can maintain populations without additional immigration. Based on the known occurrence of species, a series of efforts over the past decade has focused on developing computational approaches to approximating this ecological niche. This body of research has demonstrated that ecological niches of a species, as modelled in these frameworks:

(i) are highly predictive of distributional phenomena (Peterson et al., 2002);

(ii) permit visualization of distributions in ecological and geographical dimensions (MartínezMeyer et al., 2004);

(iii) are highly conserved over ecological and evolutionary time periods; and

(iv) constrain the long-term geographic potential of the species.

It is highly desirable that the computation of the HS should be performed at medium resolution $(\sim 1 \mathrm{~km})$ because a coarse resolution might lose detail, while a fine one is likely to introduce a bias. It is also important that the selected algorithm provides a good description of the contribution of the different climate factors to the HS. Some methods already in use, for example Algorithm Rule-Set Prediction (GARP), are black boxes that cannot provide information about the ecological processes governing the effects of the climate in this case. Without this information we can only produce "guestimates", never a complete understanding of the relationships between ticks and climate.

Baseline assessments of the HS are performed with the prevailing climate conditions and used to describe existing ecological conditions. We selected three tick species of special interest for human health in Spain, i.e. Dermacentor marginatus, Hyalomma marginatum, and Rhipicephalus turanicus since there is a substantial body of references underlining the importance of these ticks and their involvement in the transmission of prominent tickborne diseases to humans (summarized by Gilot, 1985). Also, some of these species prefer warm and dry climates and are thus good candidates for exam- ination with regard to the expected greenhouse effect, or are commonly introduced into Spain from Africa by migratory birds, e.g. H. marginatum, contributing to growing number of emerging or reemerging diseases. This is not an exhaustive list since, rather than preparing a comprehensive review of the tick problem in Spain, the intent is to provide the reader with a framework and the steps necessary to compute the process of tick infestation. Figure 1 includes the baseline HS in the Iberian Peninsula for
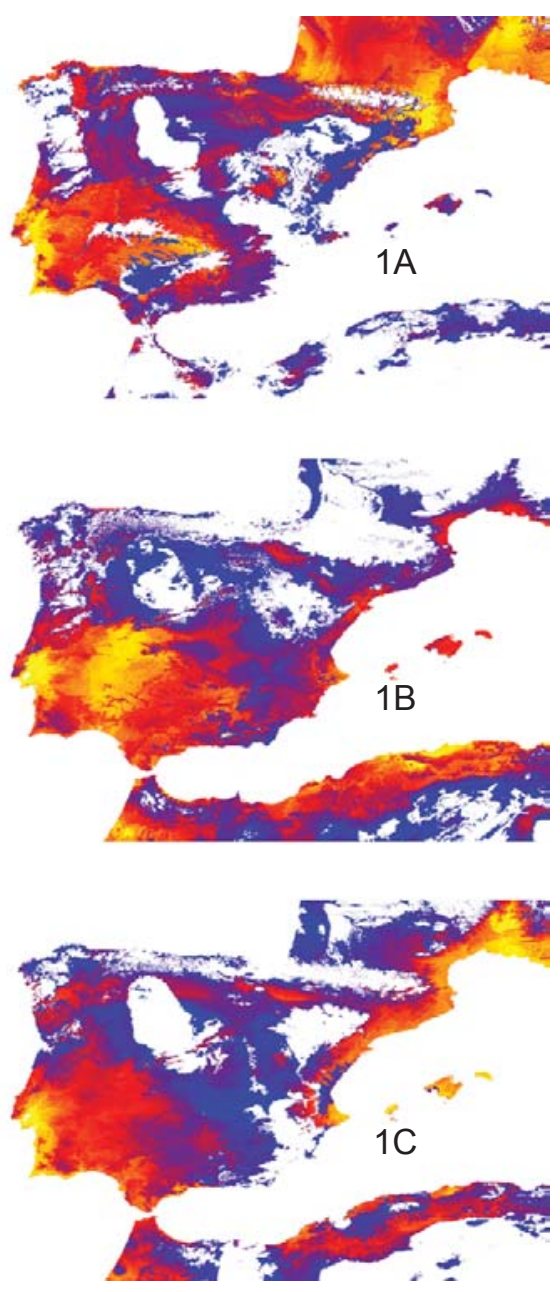

Fig. 1. The habitat (climate) suitability (HS) for the three tick species considered in the current study. A: Dermacentor marginatus; B: Hyalomma marginatum; C: Rhipicephalus turanicus. The different levels of predicted HS are shown in a scale from blue (minimum suitability) to yellow (maximum). Administrative borders correspond to the different regions of Spain. 
the three species mentioned. The HS has been calculated using MaxEnt, a maximum entropy algorithm (developed by Philips et al., 2006) based on a dataset of monthly maximum and minimum temperatures and total rainfall at a spatial resolution of $1 \mathrm{~km}$. The aspect of distribution ranges of ticks in Spain is beyond the purpose of this paper and has been considered elsewhere (Estrada-Peña and Venzal, in press).

Models, whatever the mechanism they are based on, must be tested for efficiency in predicting the tick distribution over a given territory. This is called the validation step. We assessed the performance using both presences and pseudo-absences. The evaluation of performance measures first required the derivation of so called matrices of confusion that identify true positives, true negatives, false positives and false negatives. Sensitivity is based on the concept of true-presences misclassification and is calculated as 1-percentiles of false negatives (Cumming, 2000). Sensitivity is defined as the proportion of true positives correctly predicted, whereas specificity is the proportion of true negatives correctly predicted (Fielding and Bell, 1997; Pearce and Ferrier, 2000). Threshold-independent measures, e.g. receiver operating characteristics (ROC), are considered to be more robust and more objective than threshold-dependent measures (e.g. Kappa statistics) since they do not rely on a single threshold to distinguish between predicted presence and predicted absence. From the confusion matrix we calculated the area under the curve (AUC) of a ROC plot of sensitivity against (1-specificity) (Swets, 1988). We obtained AUC from a customised function in S-plus software.

The objective of performing a baseline assessment is to calculate the area at one point in time where an evaluation species has a probability to survive greater than zero. The results of baseline assessments provide a reference point from which health authorities can:

(i) compare existing conditions in several areas in order to define management capabilities or as a guide to future planning;

(ii) predict and compare changes that may occur; and (iii) design monitoring studies.
By identifying areas where ticks are prone to exist, baseline assessments play a critical role in tick-borne disease planning. The baseline HS may be compared directly if the assessment is designed to compare existing conditions in two or more areas. Additional calculations (see below) are required if the baseline data are used as a reference point to asses the impact under scenarios of climate change.

\section{$H S$ sensitivity to climate change}

After the HS has been computed for each pixel of the target territory, it is of interest to evaluate the sensitivity of every pixel to climate changes. Sensitivity is defined as the amplitude of change in the HS with the same amplitude of climate change, e.g. if the yearly average temperature increases with $1^{\circ} \mathrm{C}$, the HS may increase up to $30 \%$ in one site, while it may swell with as much as $80 \%$ in another. This happens because different ecological conditions prevail in different zones and, again, upon the concept of $\mathrm{n}$-dimensional hyper-volume of the ecological niche. A tick population may be colonizing an area close to the core of its ecological niche where climate changes are buffered and thus have little or no influence on the HS. On the other hand, the tick population may be living in a region located in the periphery of its ecological niche and suffer considerably more because even small changes in a climate variable could produce large HS variations here. The calculation of sensitivity is important because it informs health authorities about the dimension of change to be expected in the case of a variation in climate in a given direction and magnitude. Sensitivity can be expressed according to Corson (2004) as a standard formula:

$$
S=\frac{\left(O_{H}-O_{L}\right) / O_{M}}{\left(I_{H}-I_{L}\right) / I_{M}}
$$

where $I_{H}$ is the higher value of the input parameter, $I_{L}$ the lower value of the input parameter, $I_{M}$ the 
mean of the input values, $O_{H}$ the corresponding output for the higher input value, $O_{L}$ the corresponding output for the lower input value, and $O_{M}$ the mean of the two output values.

Two approaches, top-down and bottom-up, have been recommended for the analysis of the likely impact of climate change (Parry and Carter, 1998). Many authors have used climate change scenarios generated by general circulation models as a means of investigating the likely impact of vector-borne diseases. The alternative approach, as used here, relies on sensitivity analysis of a range of climate variables. In this communication, we have used five temperature scenarios, one involving current monthly conditions, the others investigating increases and decreases from that value by $1^{\circ} \mathrm{C}$ or $2^{\circ} \mathrm{C}$. We have also modelled five rainfall scenarios, either the current monthly rainfall or changes in monthly rainfall by $60 \%, 80 \%, 120 \%$ or $140 \%$. The combinations produced from the various sets of temperature and rainfall provide an overview of the HS and sensitivity of the three tick species investigated under estimated conditions of climate warming or cooling, and/or in relation to lower and higher rainfall.

Assessing the buman dimensions: the tick impact under different scenarios of climate change

The calculation of the impact of a tick population on humans requires some variables to be added to the HS values. Sensitivity is a measure of the biological system but human populations have no such sensitivity to changes in tick abundance; the impact is rather the social consequences of the change in tick abundance. This parameter does not only involve the abundance of ticks but also the number of people in the area and the degree of tick-people contact. Impact assessments are performed by quantifying the changes in tick habitat conditions over time (or in relation to diverse scenarios) in a given area and the degree of tickhuman contact of the resident people. The understanding of the impact due to changes can be improved by using series of climate data to construct climate scenarios in a given region. Our approach here is to use the climate scenarios proposed above to evaluate the impact by ticks under various degrees of climate change.

We propose using a simple series of ratios in the calculation of the impact as follows. For most purposes, the division of a territory into its smallest administrative divisions is sufficient for defining the geographical unit to be used in the calculations and it applies commonly to the municipalities of most countries (counties in the United States). The advantage to use municipalities is that they are administrative divisions for which a range of data necessary to compute the impact (see below) can also easily be obtained. In cases where municipalities (or counties) are too large, or of disproportionate size, the target territory may be divided into hexagons of approximately $6-10 \mathrm{~km}$ radius. The advantage of using hexagons is that many series of data are already available and ready to be fitted into the analysis.

The total population of each division is the first in the series of data to be used and this value is easily obtained from the census office of the country where the evaluation is being performed. Until recently, the spatial distribution of the resident population was commonly available only at administrative levels with arbitrary borders. However, information about which areas are actually occupied can now be inferred thanks to the new tools. i.e. areal interpolation methods. This is the transformation of geographical data from one set of boundaries to another (Weichselbaum et al., 2005). If hexagons are used, population of each administrative division can be proportionally allocated to the overlying hexagons by the routines of spatial analysis and weighted by land use and land cover. In case an adequate census for the target country is lacking, the LandScan dataset of the world's population, which is presented in a "gridded" fashion (available at http://www.ornl.gov/landscan), is a useful substitute that can also be transferred proportionally to the hexagons or to the administrative 
divisions. Figure 2 shows the difference in the spatial distribution of population in Spain using either municipalities or hexagons and allocating the population proportionally.

A second parameter of importance is the degree of urbanization. Although areas with large cities have huge populations, peoples' contact with the natural environment, where the ticks live, is small for the majority of people. Areas with small cities, on the other hand, have implicitly a rural way of life with large nature contact, a piece of information which is
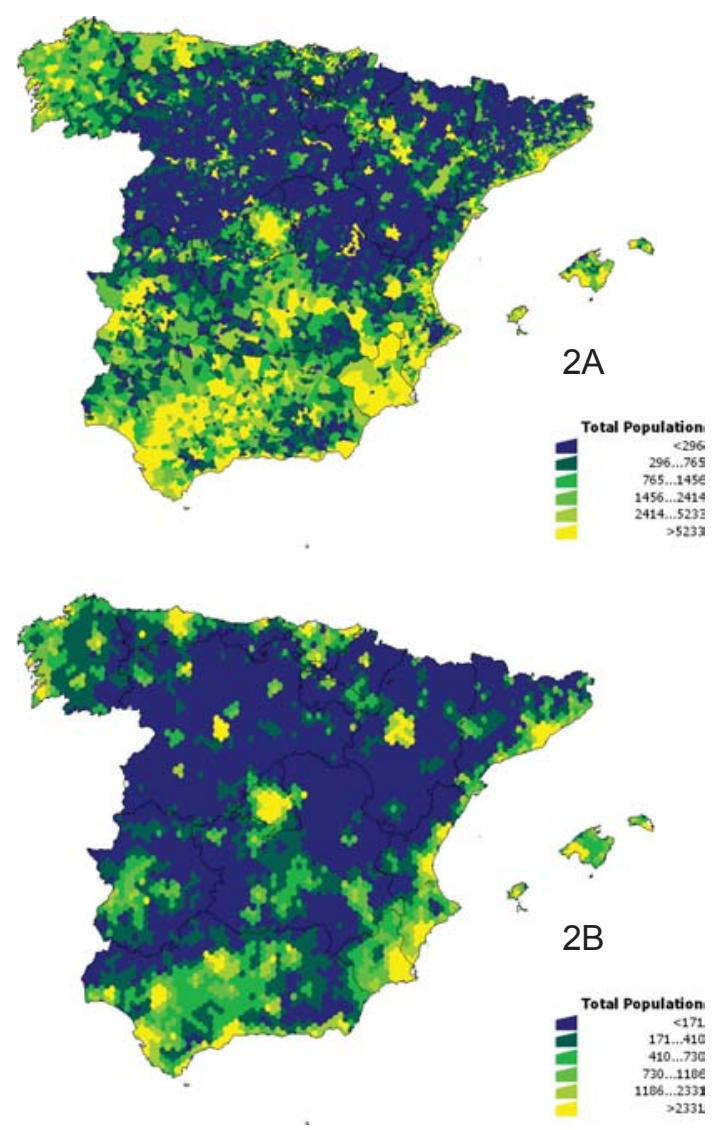

Fig. 2. A comparison of administrative versus artificial divisions used for computing the calculations and displaying the results. In A, the population distribution is shown with reference to the municipalities (administrative divisions). In B, the population values have been transferred to hexagons with a $10 \mathrm{~km}$ radius using the method of spatial interpolation. evident from LandScan data sets. Additional information about population and activities can be obtained from the dominant vegetation of the hexagon. While some countries have developed accurate classification schemes of vegetation at very high resolution (i.e. $100 \mathrm{~m}$ in the CORINE database of the European Union), this information does not adhere to other existing classification schemes. Homogeneous, uniform vegetation classifications exist at a resolution of $1 \mathrm{~km}$ for the entire world (available at http://edcwww.cr.usgs.gov). We have adhered to the Olson Global Ecosystems scheme (Olson, 1994) because of the accurate classification of biotopes and the high number of categories available. The dominant vegetation, the degree of fragmentation, and the percent of coverage of dominant vegetation are powerful indicators of the land use in each hexagon: urban, rural (agriculture, farming) or wildlife (i.e. forests).

The accessibility of the area depicted by the hexagon also needs to be included into the impact calculation. This set of data refers to the ability of the people living in the hexagon to move through their area and also to the surrounding areas. Mobility of the inhabitants is obviously a function of the mean altitude, the slope and the ranges. Sites characterized by high slopes or located within high-altitude ranges impose difficulties of variable degrees to the movements of inhabitants and are thus not commonly visited by people (mainly only tourists and certain farmers). Again, we propose to obtain the altitude as a homogeneous data set (available at http://edcwww.cr.usgs.gov) at variable resolution. For this work, we estimated that a resolution of 1 $\mathrm{km}$ provides enough features to derive the mean and range of both altitude and slope. Figure 3 includes the features of altitude and vegetation in the territory.

According to our hypothesis, the tick impact in a given hexagon under a given climate scenario is the result of a simple equation involving the change of the HS for the tick species in question in relation to the prevailing climate, the number of people living in the hexagon, the percent of rural areas in the hexagon (as measured from the dominant vegeta- 
tion and the percent of people living in rural regions), and the mobility of the inhabitants of the hexagon (accessibility). We have used a fuzzy modelling approach based on the methods outlined by Kampichler et al. (2000). This approach uses a rule base that covers the entire variable space which means that, for any possible combination of habitat and population characteristics, a rule must be provided. In the modelling of the effects of vegetation and altitude, we have adhered to the comments by Ray and Burgman (2006). All the variables mentioned above, used to compute the effect sought, represent a qualitative interpretation of ecological ideas. In many cases, the uncertainty is a discrete
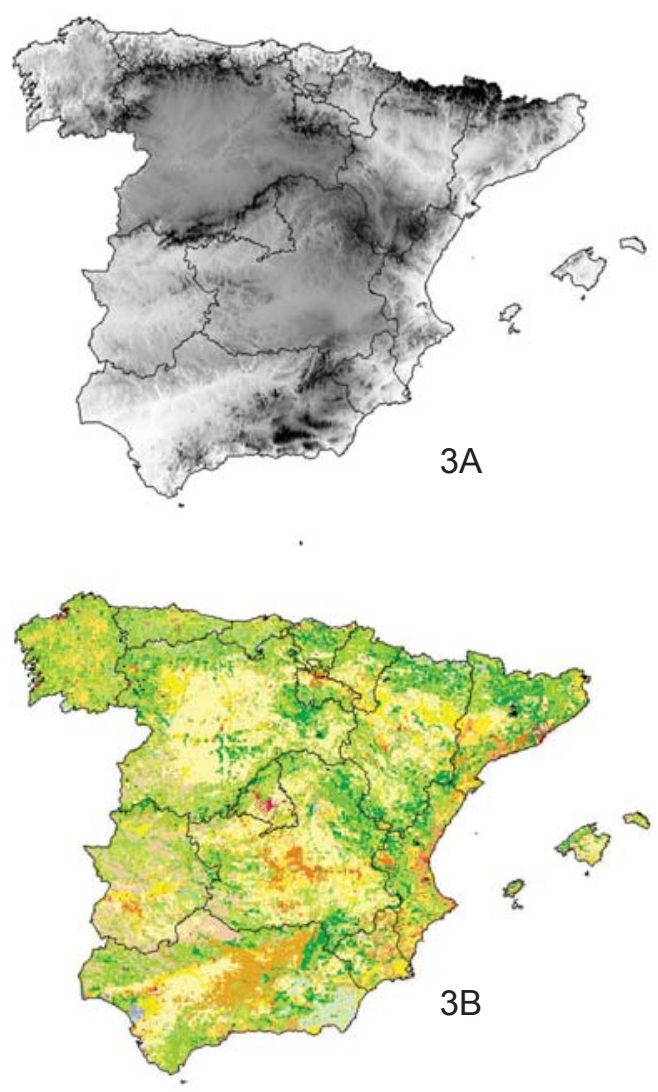

Fig. 3. Altitude (A) and natural vegetation (B) in Spain according to Olson categories of $1 \mathrm{~km}$ resolution. Higher altitudes are shown as darker. The natural vegetation is included only to provide a visual estimate of the distribution of the main vegetation categories over the territory studied. decision rather than a continuous function. Thus, we propose to handle these as "bounding maps" which can be defined as the most plausible of the set of alternative realistic choices available. The different variables used have been entered as sigmoid functions. No attempts have been done to weight each factor of the equation, i.e. to include the relative importance of people density versus the relative importance of altitude. The fuzzy functions used herein have been included in their basic forms. However, relative weighting remains a matter of much interest that should be further investigated as it could provide interesting insights into the calculation of impact of ticks on the human society under different social and geographical scenarios.

Figure 4 shows the expected tick impact on the Spanish population as a function of the climate. The outcomes of the model show that a rise in temperature together with a decrease in rainfall would have the greatest impact in the north-west of the country which is the most humid and also the coldest region in Spain (Fig. 5A). It is clear that changes in this direction would favour the invasion of this particular region by ticks as conditions would become more suitable for these parasites. Interestingly, a decrease in temperature (Fig. 5B) would produce increased tick-related problems in the eastern Mediterranean region, a site currently characterized by medium to high temperatures. This is of particular interest because this region has several heavily populated areas (Fig. 2). Both a decrease in temperature and increased rainfall would lead to a severe drop of tick impact in northwestern Spain (Figs. 5B and 5C) with an increase in the south-east, an area currently too dry to maintain significant human populations. The decreasing rainfall would have a strong positive effect on the tick populations in the north-western part of the studied territory. It is interesting to note that, according to the most prominent climate forecasts (increase of temperature and decrease of rainfall), the north-western parts of the country would suffer the highest tick pressure and hence have the highest human impact. 

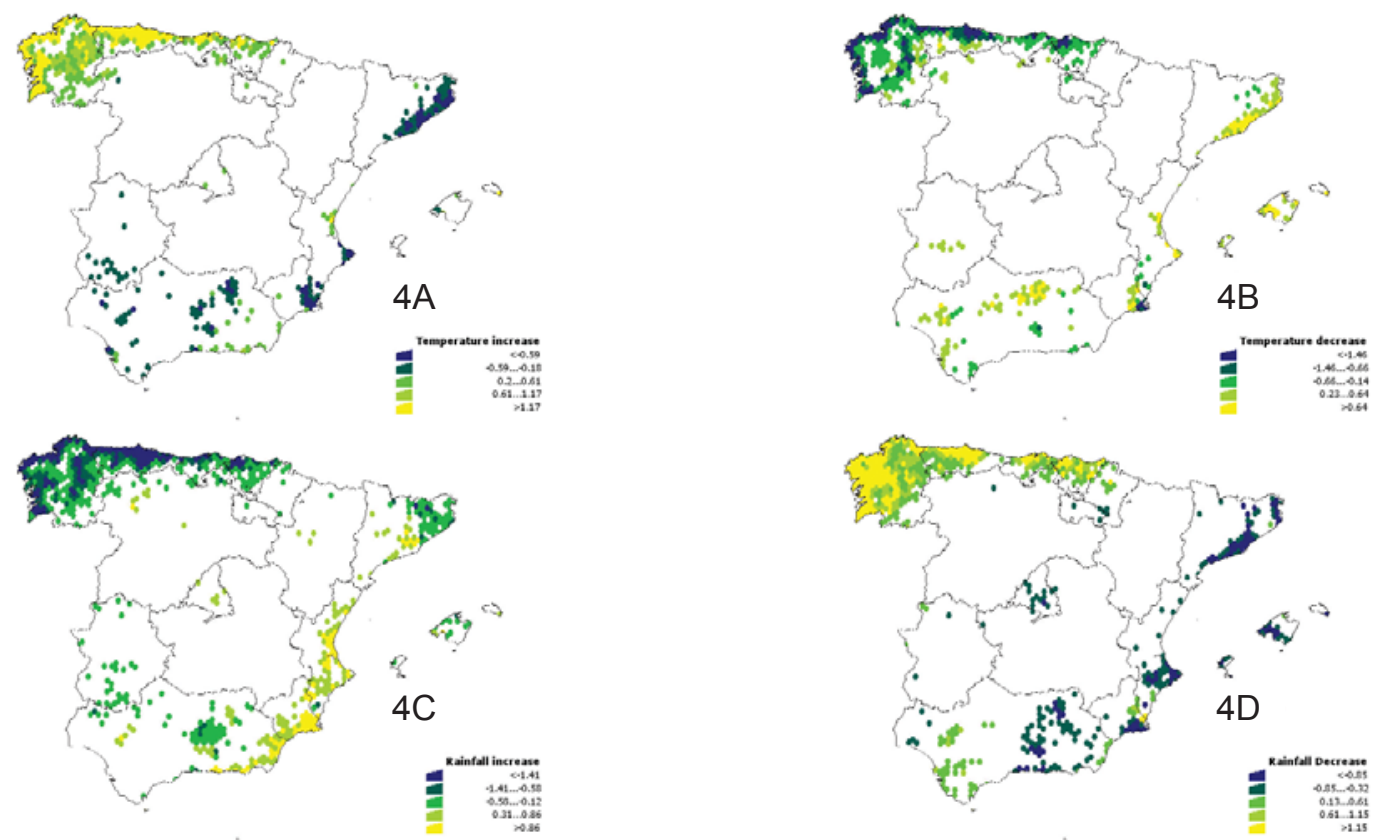

Fig. 4. The tick impact on the Spanish population in relation to four different climate scenarios. A: increase of temperature; B: decrease of temperature; C: increase of rainfall; D: decrease of rainfall. The estimated impact is depicted as calculated by a fuzzy logic interpolator. The lighter colours correspond to a decreased impact, while darker tones mean an increased impact.
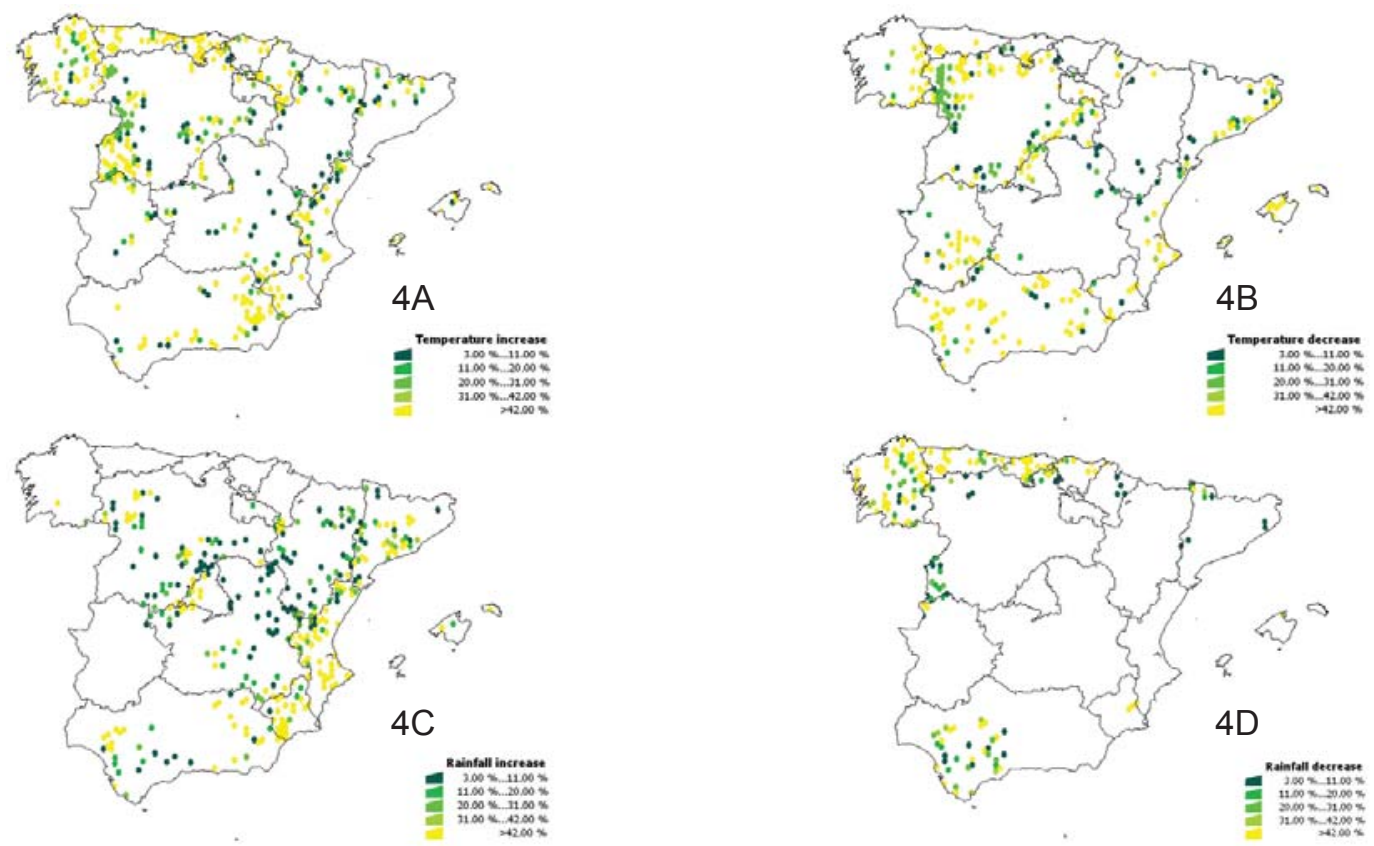

Fig. 5. The vulnerability to changes in tick populations in response to different climate scenarios. The darker colours correspond to a higher vulnerability. A: increase of temperature; B: decrease of temperature; C: increase of rainfall; D: decrease of rainfall. 


\section{The response to the problem: computing vulnerability}

The disease vulnerability is defined as a function of the presence of an infection and the host response. Vulnerability is thus a measure of the potential impact of a given change, taking into account the adaptive capacity that is available to the affected change to respond to that change. In the case of human disease, the vulnerability is the ability of the social fabric of people to respond to and manage a situation created by the introduction or increase of infectious pressure. The result could avoidance of zones with increased risk for ticks or the use of sanitary services to relieve a disease already established (either the simple tick bite or the tick-borne pathogen). Such measures should be calculated as a cost, not only in terms of direct monetary expense but also in terms of the indirect costs involved in moving to another area to perform the same activity there. Because of the need to measure and evaluate subjective costs between different countries, or even within territories of the same country, this is one of the most difficult parts of this work. A unique, comparable index that can be computed using data homogeneously available for every country is required. We propose to approach this by producing buffer zones of $10 \mathrm{~km}$ widths around each hexagon used as the main territory division and populating these buffer zones with data about accessibility (using altitude and slope), land use, and the tick impact obtained from the different climate scenarios. The vulnerability of each hexagon will thus consist of two components: the changes in the environment (expressed as the change of tick pressure) and the sanitary response (expressed as the response to the impact on human health by tickborne diseases). In the first case, the environment, vulnerability means the degree of departure from the "standard" or long-term conditions under the current climate (as observed in the hexagon) compared with those of the buffer zones (scenarios). Vulnerability in this context means the changes that the user of the land (farmer, tourist, etc.) would experience in terms of land use and tick pressure.
Null environmental vulnerability occurs when the user finds the same accessibility, the same land use and the conditions of tick pressure in both the surrounding buffer zone and in the hexagon. In other words, the "user" does not experience any change (hence, no vulnerability) if the accessibility is the same inside the buffer zone and inside the surrounded hexagon. Thus, the land use is not significantly different and the tick impact in this scenario remains within the same limits as currently found. For a given scenario of change, environment vulnerability is the net difference (between values for buffer zones and hexagons) with regard to the HS for a tick species, the accessibility and the percent of dominant land category in both "territories".

The second factor of vulnerability is the territory's sanitary response to the problems derived from ticks or tick-borne diseases. This is a complex factor because it usually involves the calculation of sanitary costs which vary considerably between countries. In addition, costs can change enormously depending on what is involved (i.e. simple diagnosis versus molecular biology approaches) and because the uncertainties of the model (the prediction of an increase of the HS is in no way a prediction of an increase of tick-borne diseases). In the context proposed here, we aim to find a simple index that can relate the sanitary structure of a country with the magnitude of the tick problem in general terms. To achieve this, we have used the number of beds of the different hospitals in Spain as a measure of the "sanitary strength" of the country. The distance of each hexagon to the closest hospital is weighted with the number of beds in that hospital as an indicative of the cost for a person suffering a tick-derived problem at a given distance of a hospital with a given number of beds. This ratio is further weighted with the total population living in the surroundings of every hospital and used as a measure of the sanitary load of a given hospital.

Taken together, these two indices provide an estimate of the "cost" that a climate scenario would produce for a person living in a given area as a result of the displacement from another area (using acces- 
sibility and vegetation as indicators). This reasoning takes into account the sanitary structure, i.e. the vulnerability which depends on living near or far away from an adequate sanitary centre. While we have seen how the potential impact is clearly concentrated in some regions (as it is a consequence of phenomena with a geographical pattern) the social structure of the vulnerability shows up as "patches" where the vulnerability is dramatically higher. The presence of such areas are commonly generated by wide differences in social activities and characterized by inadequate penetration of sanitary networks resulting in high tick loads. While the study of the impact has a great importance for the epidemiologist, the human health manager's priority is to highlight areas where the sanitary structure is inadequate and where HS favours the growth of tick populations. Therefore, although we lack reliable cost evaluations in terms of monetary resources, we have an index that provides a means to compare the vulnerability with regard to ticks in a more objective way than previously.

Figure 5 summarizes the vulnerability over the Spanish territory according to tick species and climate scenario. As shown before, both the HS and the tick impact have a clear geographical pattern reflecting both the distribution of ticks as modulated by climate or social relationships which involve tick/people contacts. However, vulnerability has no such apparent pattern because it is a measure reflecting the ability of the adaptive ability. The darker areas in Figure 5 symbolize sites with high vulnerability because the changes expected as a consequence of the different climate scenarios and the expected changes in the distribution and abundance of ticks.

\section{Limitations of the framework hypothesis}

The use of models in the management of human health is not without danger, neither for the health managers, nor for the modellers themselves. Clearly, people who have little or no training in modelling cannot easily spot limitations, uncertainties, omis- sions and subjective choices of the model. The risk is then that too much is read into the predictions of the model. There is also a danger that a model is used for purposes different from those intended, rendering invalid conclusions very likely. Our current purpose is to find a methodology capable of application in very different or geographically separate zones and develop this approach using the same set of primary data with the aim of arriving at a baseline that can be used to compare all results found. Thus, we are looking for homogeneity of results, not for a response to local problems. A framework aspiring to be of general utility must avoid parameters that can be applied only in definite regions or to specific social behaviour. Although the proposed methodology may not have the ability of "fine-tuning" when applied to local problems, it emerges as a generalpurpose tool capable of providing comparable results when applied to different geographical areas.

As every adaptation of real systems into a model, the proposed framework has limitations. A major factor is the uncertainty which is not only due to incompleteness regarding how climate and pathogens interact but also related to the complexity inherent in all human/nature systems. Moreover, few longitudinal databases provide time-frames long enough to study the type of trends required when monitoring health effects of climate change. Such models have particularly been tried for the study of climate effects on malaria (e.g. Martens, 1998; Rogers and Randolph, 2000) and of Lyme disease in the United States, the only vector-borne diseases for which adequate models and surveillance data of sufficient resolution are available (Brownstein et al., 2003). Although the knowledge of tick systems is evolving, it may only be the tip of the iceberg that has emerged so far. For example, the resilience of an ecosystem is the magnitude of a disturbance that can be absorbed or buffered by a system without the system undergoing a fundamental change or reconfiguration (Holling, 1973). Decreased resilience tends to encourage emerging diseases. We have tried to objectively compute this factor as the "sensitivity" or the "strength of change" in the tick ecosystem as a 
response to changes in climate. We have simply ignored how changes in the HS correlate with tick abundance. Some previous experiences have proven that HS has a good correlation with the probability for tick populations to persist (Estrada-Peña et al., 2006) but that tick abundance is also a property of landscape configuration (Estrada-Peña et al., 2004). Although HS and sensitivity, as computed here, are objective measurements of a property, we ignore how much the ecosystem must change before these changes can be observed as an increase or decrease of tick prevalence. Therefore, there is a pressing need for research in the last link of the chain: the actual correlation between changes in HS and changes in tick prevalence.

Impact calculations constitute a second focus of possible criticism. Tick populations may be prevalent in sites where no contact exists between the pathogen and humans nullifying the impact. In the discussion above, we have tried to quantify the degree of contact. The impact is thus an objective measure of tick exposure, which is related to the total number of people, to the ratio of population involved in countryside activities and to the accessibility of a site. A convenient way to compute the impact is to use a fuzzy function maximizing every parameter. Furthermore, the universality of the parameters evaluated makes it easy to compute the impact for any part of a continent. The sensitivity of the "impact parameter" to changes of the variables involved need to be further investigated and is also a strong focus of ongoing research. Finally, the social aspects of vulnerability are difficult to measure, as many factors are derived from habitat changes, for example, the lack of tourists in an area where the number of ticks has dramatically increased is hard to compute in a homogeneous way for every region.

What is proposed here is an approach to the computation of vulnerability but further refinements coming from the field of social sciences should be expected. To be useful and providing additional improvement of the calculations of this parameter, these refinements should focus on the consequences of change in land use and the many facets of vulnerability. The aim of this study is to produce a series of homogeneous indices of applicability to different regions, countries, or continents, as a baseline measure of tick impact. It is expected that additional performance can be attained without affecting the basic purpose of comparability.

\section{References}

Brownstein JS, Holford TR, Fish D, 2003. A climate-based model predicts the spatial distribution of Ixodes scapularis in the United States. Environ Health Perspect 111, 1152-1157.

Corson MS, 2004. Microclimate influence in a physiological model of cattle-fever tick (Boophilus spp.) population dynamics. Ecol Modell 180, 487-514.

Cumming GS, 2000. Using between-model comparisons to fine-tune linear models of species ranges. J Biogeogr 27, 441-455.

Estrada-Peña A, Bouattour A, Camicas J-L, Guglielmone AA, Horak I, Jongejan F, Latif A, Pegram R, Walter A, 2006. The known distribution and ecological preferences of the tick subgenus Boophilus (Acari: Ixodidae) in Africa and Latin America. Exp Appl Acarol 38, 219-235.

Estrada-Peña A, Martínez JM, Sánchez Acedo C, Quilez J, Del Cacho, E, 2004. Phenology of the tick Ixodes ricinus in its southern distribution range (central Spain). Med Vet Entomol 18, 387-197.

Estrada-Peña A, Venzal JM, 2007. Ecological niches of tick species in the Mediterranean region: modelling of occurrence data, distributional constraints, and impact of climate change. J Med Entomol, in press.

Fielding AH, Bell JF, 1997. A review of methods for the assessment of prediction errors in conservation presence/absence models. Environ Conserv 24, 38-49.

Gilot B, 1985. Bases biologiques, écologiques et cartographiques pour l'étude des maladies transmises par les tiques (Ixodidae et Argasidae). PhD Thesis, Université de Grenoble, 97 pp.

Hay SI, Randolph SE, Rogers D (eds.), 2000. Remote sensing and geographical information systems in epidemiology. Adv Parasitol 47, 357 pp.

Holling CS, 1973. Resilience and stability of ecological sys- 
tems. Annu Rev Ecol Syst 4, 1-23.

Kampichler C, Bartherl J, Wieland R, 2000. Density of foliage-dwelling spiders in field margins: a simple, fuzzyrule based model. Ecol Modell 129, 87-99.

Martens WJM, 1998. Climate change and vector-borne diseases. In: Health and climate change: modelling the impacts of global warming and ozone depletion. Earthscan Publications Ltd, London, 27-80 pp.

Martínez-Meyer E, Peterson AT, Navarro-Sigüenza AG, 2004. Evolution of seasonal ecological niches in the Passerina buntings (Aves: Cardinalidae): Proc Biol Sci 271, 1151-1157.

Olson JS, 1994. Global ecosystem framework-definitions: USGS EROS Data Center Internal Report, Sioux Falls, SD, $37 \mathrm{p}$.

Parry M, Carter T, 1998. Climate impact and adaptation assessment. Earthscan, London, 26 pp.

Pearce J, Ferrier S, 2000. Evaluating the predictive performance of habitat models developed using logistic regression. Ecol Modell 133, 225-245.

Peterson AT, Ball LG, Cohoon KC, 2002. Predicting distribu- tions of Mexican birds using ecological niche modelling methods. Ibis 144, 27-32.

Philips SJ, Dudík M, Schapire RE, 2006. A maximum entropy approach for species distribution modeling. Proceedings 21st Int Conf of Machina Learning.

Polley L, 2005. Navigating parasite webs and parasite flow: Emerging and re-emerging parasitic zoonoses of wildlife origin. Int J Parasitol 35, 1279-1294.

Ray N, Burgman MA, 2006. Subjective uncertainties in habitat suitability maps. Ecol Model 195, 172-186.

Rogers D, Randolph SE, 2000. The global spread of malaria in a future, warmer world. Science 289, 1763-1766.

Sutherst RW, 2004. Global change and human vulnerability to vector-borne diseases. Clin Microbiol Rev 17, 136-173.

Swets KA, 1988. Measuring the accuracy of diagnostic systems. Science 240, 1285-1293.

Weichselbaum J, Petrini-Monteferri F, Papathoma M, Wagner W, Hakner N, 2005. Sharpening census information in GIS to meet real-world conditions. The case of Earth Observation. Internal Report ESA/ESRIN Contract 18713/05/I-LG. 